\title{
Interval mapping of quantitative trait loci in an $F_{2}$ population
}

\author{
Z. W. LUO* \& M. J. KEARSEY† \\ Department of Genetics, School of Biological Sciences, The University of Birmingham, Edgbaston, \\ Birmingham B15 2TT, UK
}

\begin{abstract}
This paper attempts to provide a simple explanation of the principles of interval mapping for locating and mapping QTL. It illustrates the procedures through computer simulation and also identifies problems associated with the approach.
\end{abstract}

Keywords: interval mapping, QTL, RFLPs.

\section{Introduction}

Quantitative, continuous variation is of central importance in animal/plant breeding, medicine and evolution. This kind of variation is mainly due to the segregation of many Mendelian loci of small effect plus a major influence of the environment and thus, such variation has conventionally been studied by the statistical techniques of quantitative genetics (Mather \& Jinks, 1982; Falconer, 1989). Many attempts have been made to dissect polygenic systems using a combination of chromosome substitution techniques and genetic markers (Breese \& Mather, 1957, 1960; Thoday, 1961; Shrimpton \& Robertson, 1988a, b). However, these techniques have been restricted by the disadvantages of the morphological markers commonly used at that time.

The discovery of ubiquitous molecular genetic markers such as restriction fragment length polymorphisms (RFLPs), oligonucleotide polymorphisms (OPs) and isozymes has, for the first time, provided geneticists and animal/plant breeders with ideal markers for mapping and following the individual genes underlying the continuous variation (Botstein et al., 1980; Beckmann \& Soller, 1986; Beckmann, 1988). Interest has focused on three aspects: (i) the design of experiments to detect marker-QTL linkage (Jayakar, 1970; Hill, 1975; Luo, 1989); (ii) the relative experimental power of these designs (McMillan \& Robertson, 1974; Soller \& Brody, 1976, 1978); (iii) the experimental estimation of marker-QTL linkage (Weller, 1986; Luo \& Kearsey, 1989, 1991).

\footnotetext{
*Present address: Institute of Animal Physiology and Genetics Research, Roslin, Edinburgh EH25 9PS, UK.
}

†Correspondence: M. J. Kearsey.
Recently, Lander and his colleagues (Lander \& Botstein, 1986a, b, 1987, 1989; Lander et al., 1987) proposed the application of log-odds (LOD) which have been well used in the study of linkage in human pedigree analysis. In particular, they developed the maximum likelihood method of so-called interval mapping' to calculate the likelihood of a putative gene underlying a quantitative trait being located in a given chromosomal interval flanked by genetic markers. As a saturated genomic map of RFLPs is becoming increasingly available for many plants/animals as well as man, it is now feasible to search the entire genome for genes governing a quantitative trait and hence enable the QTL to be located systematically.

Interval mapping has been seen as a major breakthrough in the genetic analysis of polygenic traits and it has been successfully applied to dissect the polygenes controlling quantitative traits in the domestic tomato (Paterson et al., 1988, 1991). In their pioneering paper, Lander \& Botstein (1989) briefly described the main idea and algorithm of this technique for analysing experimental data from a backcross design. Furthermore, Lander and his colleagues (1987) described a FORTRAN computer program designed to map the markers and locate the QTL. However, the approaches and the relevant program have been widely considered by plant/animal breeders as being difficult to understand and this has hindered the efficient use of the method. The aims of the present paper are to elaborate the analysis and relevant algorithm of the method by examining the technique when applied to the analysis of $F_{2}$ data and also to demonstrate some limitations of its application. Finally computer simulation experiments are used to demonstrate and support the algorithm. 


\section{Theory}

\section{Experimental design and assumptions}

Consider a breeding programme starting from two inbred lines $P_{1}$ and $P_{2}$. We assume that the alleles $(Q)$ increasing the character are fixed in $P_{1}$ and the decreasing alleles $(\mathrm{q})$ in $\mathrm{P}_{2}$. If $s$ co-dominant genetic markers (for example RFLPs) are identified in the genome, then their allelic distribution among the two parents is defined such that the marker alleles in $P_{1}$ are denoted by $M$ while the alleles in $\mathrm{P}_{2}$ are denoted by $m$. Therefore, there are three genotypes for each quantitative trait locus (QTL) and each marker gene respectively. The $F_{2}$ family is produced from selfing or sibmating the $F_{1}$ between $P_{1}$ and $P_{2}$. By using conventional methods one can set up a marker linkage map in which the genome is separated into a series of chromosomal segments (intervals), each of which is flanked by two marker loci. If a QTL exists in the $i$ th interval, its location can be summarized as follows.

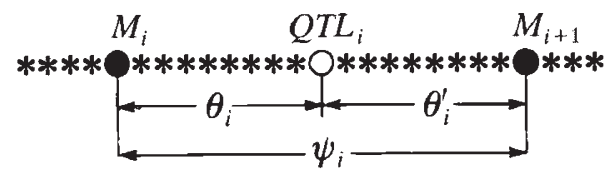

Here $\psi_{i}$ is the map distance between the two marker loci, which can be obtained directly from the linkage map, while $\theta_{i}$ and $\theta_{i}^{\prime}$ are mapping distances from the QTL to its left and right markers respectively. They follow the well-known mapping function of Haldane (1919):

$\psi_{i}=\theta_{i}\left(1-\theta_{i}^{\prime}\right)+\theta_{i}^{\prime}\left(1-\theta_{i}\right)$

where $\theta_{i}^{\prime}$ can be solved for a known $\psi_{i}$ and a given $\theta_{i}$ as

$\theta_{i}^{\prime}=\frac{\psi_{i}-\theta_{i}}{1-2 \theta_{i}}$

\section{The EM algorithm in QTL interval mapping}

Interval mapping a QTL by the above model involves two steps: the first is to detect the presence of the QTL and the second is to locate it once its presence has been confirmed. However, these two are not independent. The first step is completed by testing the likelihood of the hypothesis that there is a QTL at a given site in the interval against the likelihood that the hypothesis is not true. The logarithm of the ratio of these two likelihoods, i.e. LOD score, is used as a measure of the reliability of the hypothesis. Once the LOD score is higher than a given threshold the hypothesis is accepted, otherwise it is rejected. By scanning all possible sites throughout the interval, one can obtain corresponding LOD scores distributed over the interval, and the site at which the LOD score has its maximum value will be the most likely location of the QTL.

In the $F_{2}$ family discussed previously, a sample of $n$ individuals is scored for their quantitative phenotype, denoted by $Y=\left\{y_{1}, y_{2}, \ldots, y_{n}\right\}$, and their marker genotype. The effects of the three genotypes $(\mathrm{QQ}, \mathrm{Qq}$ and qq) of a particular QTL are assumed to be $N\left(\alpha_{1}, \sigma^{2}\right)$, $N\left(\alpha_{2}, \sigma^{2}\right)$ and $N\left(\alpha_{3}, \sigma^{2}\right)$, respectively. These distribution parameters may vary for different QTL. The distribution of effect of the QTL on the quantitative character will be a mixture of three normal distributions with frequencies denoted by $p_{1}, p_{2}$ and $1-p_{1}-p_{2}$ respectively. Its probability density function can be written as

$$
\begin{aligned}
f_{Y}(y \mid \phi)= & p_{1} u\left(y-\alpha_{1}\right)+p_{2} u\left(y-\alpha_{2}\right) \\
& +\left(1-p_{1}-p_{2}\right) u\left(y-\alpha_{3}\right)
\end{aligned}
$$

where $\phi=\left(p_{1}, p_{2}, \alpha_{1}, \alpha_{2}, \alpha_{3}, \sigma^{2}\right)^{T}$ represents the vector of unknown parameters and

$u(z)=\frac{1}{\sqrt{2 \pi \sigma^{2}}} \exp \left(-\frac{z^{2}}{2 \sigma^{2}}\right)$.

In order to isolate individual genotypic effects of the putative QTL, one needs to estimate the unknown parameter vector $\phi$. This is statistically equivalent to resolving the mixed distributions. The maximum likelihood estimate of $\phi$ can be obtained by using the well known EM algorithm developed by Dempster et al. (1977). Given a QTL flanked by two markers, there will be nine different gametes and $27 \mathrm{~F}_{2}$ genotypes. The expected frequencies of these genotypes, $\bar{\omega}_{j k}^{(0)}\left(j=1,2, \ldots, n, k=1,2,3\right.$ for $F_{2}$ population), in the $i$ th interval are as shown in Table 1 . Based on these frequencies and the observations of the quantitative character, an initial estimate of the unknown parameter, $\phi$, can be derived from the following formulae (Hosmer, 1973; Louis, 1982; Leytham, 1984; Little \& Rubin, 1987):

$$
\begin{aligned}
& p_{k}^{(0)}=\frac{1}{n} \sum_{j=1}^{n} \bar{\omega}_{j k}^{(0)} \\
& \alpha_{k}^{(0)}=\frac{1}{n p_{k}^{(0)}} \sum_{j=1}^{n} \bar{\omega}_{j k}^{\langle 0\rangle} y_{j} \\
& \sigma^{(0)^{2}}=\frac{1}{n}\left[\sum_{k=1}^{3} \sum_{j=1}^{n} \bar{\omega}_{j k}^{\langle 0\rangle}\left(y_{j}-\alpha_{k}^{(0)}\right)^{2}\right] .
\end{aligned}
$$

The EM algorithm starts with these initial estimates as follows. The expectation step (E-step) uses the initial 
Table 1 The probability of the three QTL genotypes in an $\mathrm{F}_{2}$ population for a given flanking marker genotypes*. $A=\theta \theta^{\prime}+(1-\theta)\left(1-\theta^{\prime}\right)$ and $B=\theta\left(1-\theta^{\prime}\right)+\theta^{\prime}(1-\theta)$.

QTL genotypes

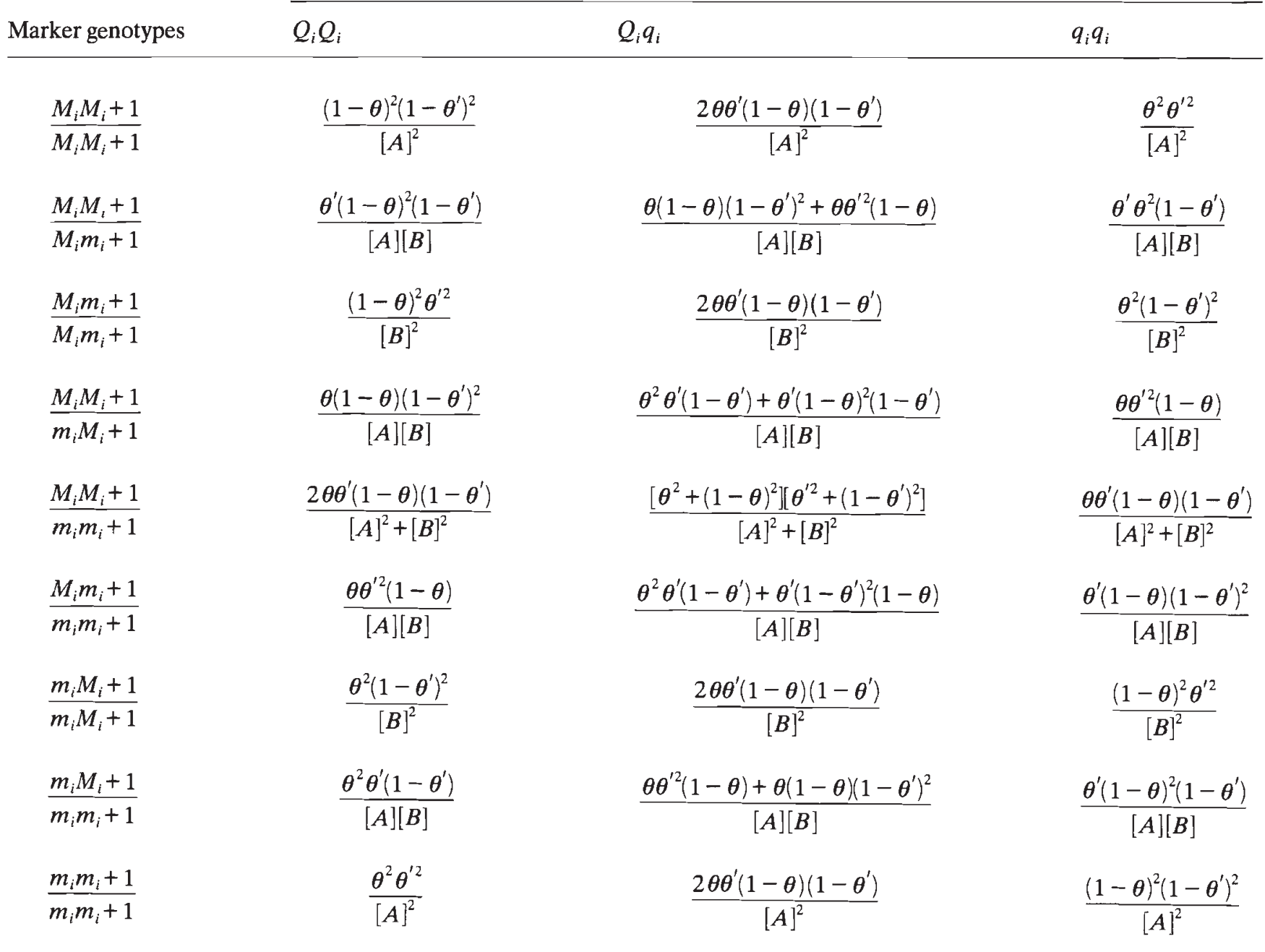

estimates $p_{k}^{(0)}, \alpha_{k}^{(0)}$ and $\sigma^{(0)^{2}}$ as if they were the true parameter values and computes the new

$$
\bar{\omega}_{j k}^{(1)}=\frac{p_{k}^{(0)} u\left(y_{j}-\alpha_{k}^{(0)}\right)}{\sum_{k=1}^{3} p_{k}^{(0)} u\left(y_{j}-\alpha_{k}^{(0)}\right)} .
$$

The maximization step ( $M$-step) is based on the new estimates $\bar{w}_{j k}^{(1)}$ to yield the following new estimates

$$
\begin{aligned}
& p_{k}^{(1)}=\frac{1}{n} \sum_{j=1}^{n} \bar{\omega}_{j k}^{(1)} \\
& \alpha_{k}^{(1)}=\frac{1}{n p_{k}^{(1)}} \sum_{j=1}^{n} \bar{\omega}_{j k}^{(1)} y_{j}
\end{aligned}
$$

$$
\sigma^{(1)^{2}}=\frac{1}{n}\left[\sum_{k=1}^{3} \sum_{j=1}^{n} \bar{\omega}_{j k}^{(1)}\left(y_{j}-\alpha_{k}^{(1)}\right)^{2}\right]
$$

which will increase the value of the following likelihood function,

$$
L\left(y \mid p_{k}, \alpha_{k}, \sigma^{2}\right)=\prod_{j=1}^{n} \frac{1}{\sqrt{2 \pi \sigma^{2}}}\left[\sum_{k=1}^{3} p_{k} \exp \left[-\frac{\left(y_{j}-\alpha_{k}\right)^{2}}{2 \sigma^{2}}\right]\right]
$$

(Hosmer, 1973; Louis, 1982; Titterington et al., 1985).

The $E$ and $M$ steps are repeated iteratively following equations (4), and (5), so that a sequence of estimates, $\left\{p_{k}^{(t)}, \alpha_{k}^{(t)}, \sigma^{(t)^{2}}\right\}_{t=1,2, \ldots}$ will be generated. Wu (1983) has 
shown that this sequence will converge to its limit at which derivative of the likelihood function (6) takes a value of zero and hence this point is a global maximum of the function, which provides the maximum likelihood estimates required. Let these estimates be represented by $\left(p_{k}^{*}, \alpha_{k}^{*}, \sigma^{* 2}\right)$, then the likelihood ratio of locating the QTL at the site of $\theta_{i} \mathrm{cM}$ away from its left marker locus $M_{i}$, is

$\operatorname{LOD}\left(\theta_{i}\right)=\log _{10}\left[\frac{L\left(Y \mid p_{k}^{*}, \alpha_{k}^{*}, \sigma^{* 2}\right)}{L\left(Y \mid 1, \alpha^{* *}, \sigma^{* * 2}\right)}\right]$

where

$\alpha^{* *}=\frac{1}{n} \sum_{j=1}^{n} y_{j}$

$\sigma^{* * 2}=\frac{1}{n} \sum_{j=1}^{n}\left(y_{j}-\alpha^{* *}\right)^{2}$

If every interval of each chromosome of the genome studied is scanned for the presence of QTL at all possible locations by the methods discussed above, a curve of LOD scores against the searched chromosomal locations will be obtained for every chromosome.

A threshold is required for statistical inference, i.e. those regions of the chromosome for which the LOD score exceeds this threshold, may be regarded as potential sites of a QTL while its most likely position is where the LOD score is maximum. Lander \& Botstein (1989) discussed general rules for determining an appropriate threshold for the LOD score following the principle of minimizing the probability of type II errors and found that the appropriate threshold depends not only on the size of the genome but also on the density of genetic markers mapped. In fact, as the number of intervals $(m)$ tends to infinity, the required nominal significance level for each individual test approaches a non-zero limit independent of $s$, the number of marker genes scored. Typically, a LOD threshold of between 2 and 3 is required to ensure a false-positive ratio of 5 per cent.

\section{Computer simulation}

In this section the interval mapping algorithm developed for an $F_{2}$ population will be tested by a computer simulation experiment.

\section{The genetic background and parameters simulated}

A series of FORTRAN-77 and PASCAL computer programs have been designed to simulate the genetic behaviour of linkage among marker genes and QTL. These pro- grams allow us to simulate populations with different genetic structures to be produced by combining gametes generated from defined parents through a 'random walk' procedure as described by Crosby (1973) and Luo (1989). Any number of quantitative characters, chromosomes, marker loci and QTL, as well as different linkage relationships among markers and QTL, can be easily simulated by these programs. The programs also allow varying degrees of linkage to be accommodated between markers and QTLs as well as various dominance ratios and heritabilities (in broad or narrow sense) of the quantitative characters to be considered.

In the present paper, the QTL data published by Paterson et al. (1988) are used as a model to test the algorithm. These data came from a backcross population derived from two lines of tomato. The purpose of the original experiment was to dissect the polygenes which control three quantitative traits, i.e. fruit mass (FM); soluble solids ( $\mathrm{SS})$; fruit $\mathrm{pH}(\mathrm{pH})$, by using $67 \mathrm{co}^{-}$ dominant genetic markers. The linkage relationships between the QTL and marker loci are modelled on those published by Paterson et al. (1988) and they are illustrated in Fig. 1. We have, for ease of comparison, assigned the three quantitative characters the same broad heritability of 50 per cent and assumed equal and additive effects for all the QTL. The dominance ratio of each increasing allele is 0.5 for all QTL. The simulated experiment involved $250 \mathrm{~F}_{2}$ individuals as did the original backcross experiment on which it was based.

\section{Simulation results}

The LOD scores calculated from the simulation data are plotted against locations of the genetic markers and the results are summarized in Fig. 1 for the 12 chromosomes respectively. The abscissa of these plots is scaled by the length of the marked chromosome in centimorgans ( $1 \mathrm{cM}=1$ per cent recombination frequency), while the ordinates represent the LOD scores.

\section{Discussion}

The LOD plots for chromosomes 2, 5 and 12 consistently fail to reach the threshold 2.4 , confirming the absence of any QTL on these chromosomes. Although non-zero LOD are due to chance, they clearly have local maxima in the vicinity of the marker loci.

It is clear from the LOD plot for chromosome 1 that the curve for character 1 (FM) has an obvious mode occurring on the left arm of the chromosome, corresponding to the known positions of the three genes. However, the corresponding curves for the other two 
characters have LOD scores consistently lower than the threshold of 2.4, confirming the absence of QTL controlling these characters on that chromosome.

A comparison of chromosomes 1 and 7 on which there are 3 and 1 QTL, respectively, clearly indicates the reinforcing effects of genes linked in association on the LOD score. If we just consider those chromosomes which contain only one gene for character 1 (chromosomes 4, 6, 7 and 9), we find considerable variation both in the maximum value of the LOD score and the positions of the maximum in relation to the true position of the QTL. On chromosome 4, for example, the LOD score maximum is clearly situated at position
0.25 on the chromosome, while the gene is actually located considerably further away at 0.49 .

The LOD score trajectory for character 3 on chromosome 10 shows two modes, confirming that there are at least two QTL for this character on this chromosome. Although there is more than one QTL for character 1 on chromosome 11, for character 3 on chromosome 8 and for characters 2 and 3 on chromosome 6 , they are sufficiently close together to produce a single mode for the LOD score.

Following the main ideas of Lander \& Botstein (1989), an algorithm has been developed in the present paper to analyse an $F_{2}$ population. The emphasis was
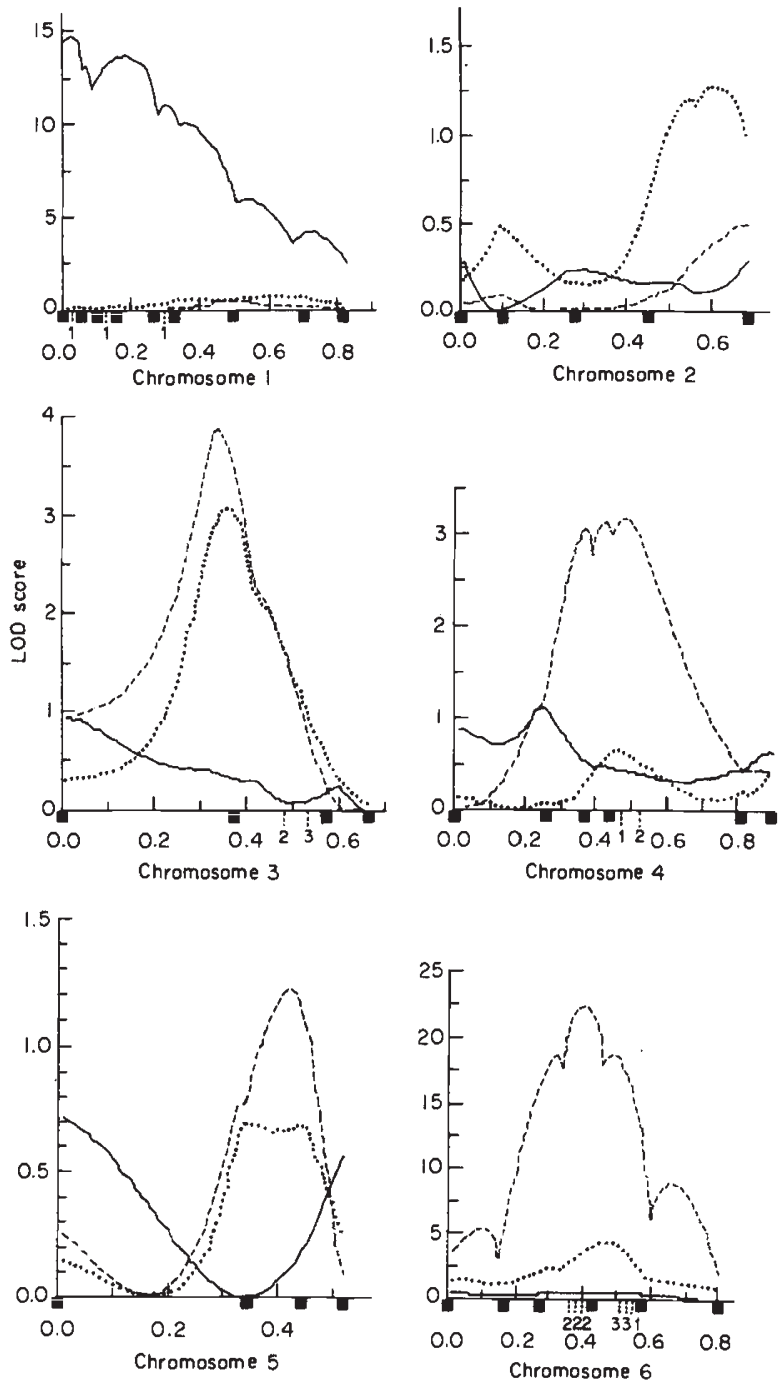
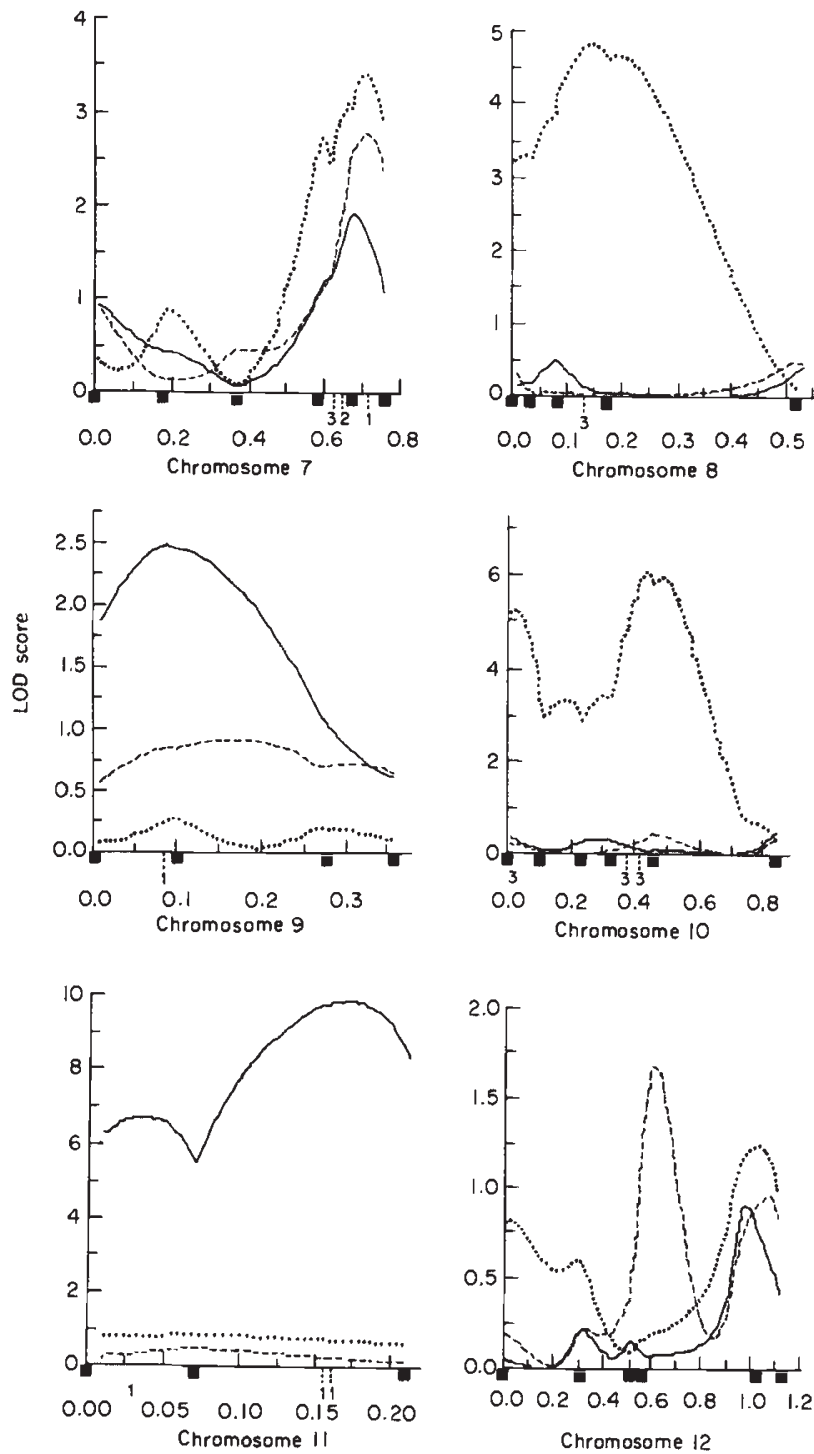

Fig. 1 The LOD distributions for the three quantitative characters on the 12 simulated chromosomes. LOD curves for characters 1,2 and 3 respectively $(-,---, \ldots)$. The dots marked on the abscissa indicate the locations of the marker genes and those integers under the abscissa represent the locations of the QTLs of the corresponding traits. The abscissa was scaled in terms of recombination frequency. 
placed on searching for the chromosomal segments which were associated with quantitative effects throughout the genome. Although these analyses can also provide estimates of the mean and variance of the QTL detected, these estimates could reflect a joint effect of several closely linked QTL.

The results of the simulation experiment show a very good agreement with the actual distributions of QTL, confirming the reliability of the algorithm and indicating that an $F_{2}$ population is as convenient to analyse as a backcross.

It has been assumed in the theory section that the three genotypes at each QTL have the same variation. This assumption can in fact be relaxed, if the equations (3.3) and (5.3) are replaced respectively by

$\sigma_{k}^{(0)^{2}}=\frac{1}{n p_{k}^{(0)}} \sum_{j=1}^{n} \bar{\omega}_{j k}^{(0)}\left(y_{j}-\alpha_{k}^{(0)}\right)^{2}$

and

$\sigma_{k}^{(1)^{2}}=\frac{1}{n p_{k}^{(1)}} \sum_{j=1}^{n} \bar{\omega}_{j k}^{(1)}\left(y_{j}-\alpha_{k}^{(1)}\right)^{2}$

where $k=1,2,3$ refer to three QTL genotypes.

Our simulation has been based on the unwarranted assumption that all alleles increasing the quantitative characters on any given chromosome are fixed in one parent while all the decreasing alleles are fixed in the other. Failure of this assumption will result in decreased efficiency of 'interval mapping'. Figure 2 displays 'interval mapping' results of two simulated $F_{2}$ populations. In each case, the simulated linkage map consists of 10 marker loci and the quantitative character studied is affected by two linked QTL. The markers and QTL are distributed on one chromosome with a length of $100 \mathrm{cM}$ and their locations are as illustrated in Fig. 2. The two simulations share the same parameters (broad heritability of 10 per cent and dominance ratio of 0.5 for the quantitative character), same population size of 100 , as well as the same random seed which implies that the chiasma events simulated are identical for the two populations. The only difference is that the first $F_{2}$ population is generated from two inbreds in which two QTL are associated, i.e. two parents have quantitative character genotypes as $++/ /++$ and $--/ /--$ respectively, while the second population is produced by two dispersed QTL parents, i.e. they have genotypes $+-/ /$ +- and $-+/ 1-+$. We have simulated these two situations many times and found that the LOD score plots were typically quite similar to those shown in Fig. 2. Thus, when associated parents were used the corresponding LOD curves exhibited two clear modes and regularly had higher values, while the LOD score

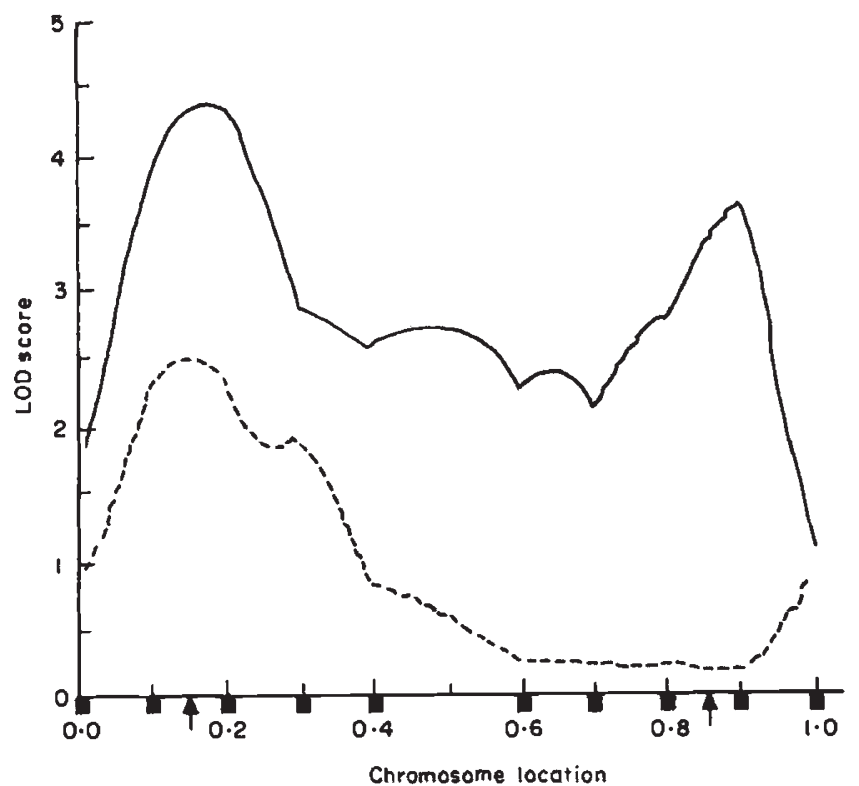

Fig. 2 Comparison between mapping associated and dispersed quantitative genes by use of interval mapping. The solid and break lines represent the LOD curves for the quantitative genes in association and dispersion respectively. The solid dots and the arrows on the abscissa refer to the locations of the marker genes and the QTL respectively. The abscissa is scaled in terms of recombination frequency.

curves for the dispersed cross usually show just one mode occurring left, centre or right while the maxima were relatively lower. Therefore, the efficiency of the methods would be limited by the distribution of QTL among the parents.

Because of the complicated genetic properties of quantitative variation, it is still a hard task for geneticists and animal/plant breeders to clarify the enormous complexities. As pointed out by Michelmore \& Shaw (1988), computer analyses would be a powerful tool, at least in theoretical investigations, to strengthen our understanding of these problems. We have successfully compiled a series of computer simulation and analysis programs (in FORTRAN-77 and PASCAL versions) for marker QTL analysis and are quite happy to make our original programs available to interested colleagues.

\section{References}

BECKMANN, J. S. 1988. Oligonucleotide polymorphisms: A new tool for genomic genetics. Biotechnology, 6, 1061-1064.

BECKMANN, J. S. AND SOLLER, M. 1986. Restriction fragment length polymorphisms in plant genetic improvement. In: Miflin, B. J. (ed.). Oxford Surveys of Plant Molecular and Cell Biology, 3, Oxford Press, Oxford, pp. 196-250.

BOTSTEIN, D., WHITE, R. L., SKOLNICK, M. AND DAVIES, R. W. 1980. Construction of a genetic linkage map in man using 
restriction fragment length polymorphism. Am. J. Hum. Genet., 32, 314-331.

BREESE, E. L. AND MATHER, K. 1957. The organisation of polygenic activity within a chromosome in Drosophila: I. Hair characters. Heredity, 11, 373-395.

BREESE, E. L. AND MATHER, K. 1960. The organisation of polygenic activity within a chromosome in Drosophila: II. Viability. Heredity, 14, 375-399.

CRosby, J. L. 1973. Computer Simulation in Genetics. John Wiley \& Sons, London.

DEMPSTER, A. P., LAIRD, N. M. AND RUBIN, D. B. 1977. Maximum likelihood from incomplete data via the EM algorithm. $J$. R. Statist. Soc. B, 39, 1-22.

FALCONER, D. S. 1989. Introduction to Quantitative Genetics, 3rd edn. Longman Scientific \& Technical, Harlow.

HALDANE, J. B. S. 1919. The combination of linkage values and the calculation of distance between the loci of linked factors. J. Genet., 8, 299-309.

HILL, A. P. 1975. Quantitative linkage: a statistical procedure for its detection and estimation. Ann. Hum. Genet. Lond., 38, 439-449.

HOSMER, D. W. 1973. A comparison of iterative maximum likelihood estimates of the parameters of a mixture of two normal distributions under three different types of sample. Biometrics, 29, 761-770.

JAYAKAR, S. D. 1970. On the detection and estimation of linkage between a locus influencing a quantitative character and a marker locus. Biometrics, 26, 451-464.

LANDER, E. S. AND BOTSTEIN, D. 1986a. Strategies for studying heterogeneous genetic traits in humans by using a linkage map of restriction fragment length polymorphisms. Proc. Natl. Acad. Sci., U.S.A., 83, 7353-7357.

LANDER, E. S. AND BOTSTEIN, D. 1986b. Mapping complex genetic traits in humans: new methods using a complete RFLP linkage map. Cold Spring Harbor Symp. Quant. Biol., 51, 49-62.

LANDER, E. S. AND BOTSTEIN, D. 1987. Homozygosity mapping: a way to map human recessive traits with the DNA of inbred children. Science, 236, 1567-1570.

LANDER, E. S., GREEN, P., ABRAHAMSON, J., BARLOW, A., DALY, M. J., LINCOLN, S. E. AND NEWBURG, L. 1987. Mapmarker: an interactive computer package for constructing primary genetic linkage maps of experimental and natural populations. Genomics, 1, 174-181.

LANDER, E. S. AND BOTSTEIN, D. 1989. Mapping Mendelian factors underlying quantitative traits using RFLP linkage maps. Genetics, 121, 185-199.

LANDER, E. S. AND GREEN, P. 1987. Construction of multilocus genetic linkage maps in humans Proc. Natl. Acad. Sci., U.S.A., 84, 2363-2367.

LEYTHAM, K. M. 1984. Maximum likelihood estimates for the parameters of mixture distributions. Water Resour. Res., 20, 896-902.

LITTLE, R. J. A. AND RUBIN, D. B. 1987. Statistical Analysis with Missing Data. John Wiley, New York.

LouIs, T. A. 1982. Finding the observed information matrix when using the EM Algorithm. J. R. Statist. Soc. B, 44, 226-233.

LUO, Z. W. AND KEARSEY, M. J. 1989. Maximum likelihood estimation of linkage between a marker gene and a quantitative locus. Heredity, 63, 401-408.

LUo, Z. W. AND KEARSEY, M. J. 1991. Maximum likelihood estimation of linkage between a marker gene and a quantitative locus. II. Application to backcross and doubled haploid populations. Heredity, 63, 401-408.

LUo, z. w. 1989. Polygene Location and Selection for Heterotic trait in Drosophila. PhD Thesis. University of Birmingham.

MATHER, K. AND JINKS, J, L. 1982. Biometrical Genetics, 3rd edn. Chapman and Hall, London.

McMILLAN, I. AND ROBERTSON, A. 1974. The power of methods for the detection of major genes affecting quantitative characters. Heredity, 32, 349-356.

MICHELMORE, R. W. AND SHAW, D. v. 1988. Character dissection. Nature, 335, 672-673.

PATERSON, A. H., LANDER, E. S., HEWITT, J. D., PETERSON, S., LINCOLN, S. E. AND TANKSLEY, S. D. 1988. Resolution of quantitative traits into Mendelian factors by using a complete RFLP linkage map. Nature, 335, 721-726.

PATERSON, A. H., DAMON, S., HEWITT, J. D., ZAMIR, D., RABINOWITCH, H. D., LINCOLN, S. E., LANDER, E. S. AND TANKSLEY, S. D. 1991. Mendelian factors underlying quantitative traits in tomato: comparison across species, generations and environments. Genetics, 127, 181-197.

SHRIMPTON, A. E. AND ROBERTSON, A. 1988a. The isolation of polygenic factors controlling bristle score in Drosophila melanogaster. I. Allocation of third chromosome sternopleural bristle effects to chromosome sections. Genetics, 118, 437-443.

SHRIMPTON, A. E. AND ROBERTSON, A. $1988 \mathrm{~b}$. The isolation of polygenic factors controlling bristle score in Drosophila melanogaster. II. Distribution of third chromosome bristle effects within chromosome sections. Genetics, 118, 445-459.

SOLLER, M. AND BRODY, T. 1976. On the power of experimental designs for the detection of linkage between marker loci and quantitative loci in crosses between inbred lines. Theoret. Appl. Genet., 47, 35-39.

SOLLER, M. AND GENIZI, A. 1978. The efficiency of experimental designs for the detection of linkage between a marker locus and a locus affecting a quantitative trait in segregating populations. Biometrics, 34, 47-55.

THODAY, M. L. 1961. Location of polygenes. Nature, 191, 368-370.

TITTERINGTON, D. M., SMITH, A. F. AND MAKOV, U. E. 1985. Statistical Analysis of Finite Mixture Distributions. John Wiley, New York.

WELLER, J. I. 1986. Maximum likelihood techniques for the mapping and analysis of quantitative trait loci with the aid of genetic markers. Biometrics, 42, 627-640.

wU, C. J. 1983. On the convergence properties of the EM algorithm. The Annals of Statistics, 11, 95-103. 